Article

\title{
Biosynthesis of Polyunsaturated Fatty Acids in Octopus vulgaris: Molecular Cloning and Functional Characterisation of a Stearoyl-CoA Desaturase and an Elongation of Very Long-Chain Fatty Acid 4 Protein
}

\author{
Óscar Monroig ${ }^{1, *}$, Rosa de Llanos ${ }^{2,3}$, Inmaculada Varó ${ }^{4}$, Francisco Hontoria ${ }^{4}$, \\ Douglas R. Tocher ${ }^{1}$, Sergi Puig ${ }^{3}$ and Juan C. Navarro ${ }^{4}$ \\ 1 Institute of Aquaculture, Faculty of Natural Sciences, University of Stirling, Stirling FK9 4LA, Scotland, UK; \\ d.r.tocher@stir.ac.uk \\ 2 School of Applied Sciences, Edinburgh Napier University, Edinburgh EH11 4BN, Scotland, UK; \\ R.DeLlanos@napier.ac.uk \\ 3 Instituto de Agroquímica y Tecnología de Alimentos (IATA-CSIC), Paterna, Valencia 46980, Spain; \\ spuig@iata.csic.es \\ 4 Instituto de Acuicultura Torre de la Sal (IATS-CSIC), Ribera de Cabanes, Castellón 12595, Spain; \\ inma@iats.csic.es (I.V.); hontoria@iats.csic.es (F.H.); jcnavarro@iats.csic.es (J.C.N.) \\ * Correspondence: oscar.monroig@stir.ac.uk; Tel.: +44-1786-467892
}

Academic Editors: Rosário Domingues, Ricardo Calado and Pedro Domingues Received: 14 February 2017; Accepted: 16 March 2017; Published: 21 March 2017

\begin{abstract}
Polyunsaturated fatty acids (PUFAs) have been acknowledged as essential nutrients for cephalopods but the specific PUFAs that satisfy the physiological requirements are unknown. To expand our previous investigations on characterisation of desaturases and elongases involved in the biosynthesis of PUFAs and hence determine the dietary PUFA requirements in cephalopods, this study aimed to investigate the roles that a stearoyl-CoA desaturase (Scd) and an elongation of very long-chain fatty acid 4 (Elovl4) protein play in the biosynthesis of essential fatty acids (FAs). Our results confirmed the Octopus vulgaris Scd is a $\Delta 9$ desaturase with relatively high affinity towards saturated FAs with $\geq C_{18}$ chain lengths. Scd was unable to desaturate 20:1n-15 ( $\left.{ }^{\Delta 5} 20: 1\right)$ suggesting that its role in the biosynthesis of non-methylene interrupted FAs (NMI FAs) is limited to the introduction of the first unsaturation at $\Delta 9$ position. Interestingly, the previously characterised $\Delta 5$ fatty acyl desaturase was indeed able to convert 20:1n-9 $\left({ }^{\Delta 11} 20: 1\right)$ to ${ }^{\Delta 5,11} 20: 2$, an NMI FA previously detected in octopus nephridium. Additionally, Elovl4 was able to mediate the production of 24:5n-3 and thus can contribute to docosahexaenoic acid (DHA) biosynthesis through the Sprecher pathway. Moreover, the octopus Elovl4 was confirmed to play a key role in the biosynthesis of very long-chain $\left(>\mathrm{C}_{24}\right)$ PUFAs.
\end{abstract}

Keywords: biosynthesis; elongation of very long-chain fatty acids 4 protein; non-methyleneinterrupted fatty acids; polyunsaturated fatty acids; Octopus vulgaris; stearoyl-CoA desaturase

\section{Introduction}

Cephalopods have been regarded as promising candidates for the diversification of marine aquaculture due to their great commercial interest [1]. Despite significant progress made over the last decade, culture of cephalopod species with pelagic paralarval stages like the common octopus Octopus vulgaris is still challenging due to the massive mortalities occurring upon the settlement phase [2]. The specific factors causing such mortalities of paralarvae remain unclear, although it has become increasingly obvious that nutritional issues associated with inadequate supply of essential 
nutrients such as lipids are crucial to ensure normal growth and development of $O$. vulgaris paralarvae and ultimately improve their viability [3].

Previous investigations postulated that polyunsaturated fatty acids (PUFAs) are essential nutrients for the common octopus [4,5]. However, the specific PUFAs that satisfy the physiological requirements were not determined, partly due to the difficulties in running nutritional trials on octopus paralarvae. In order to provide insights to the endogenous capability for PUFA biosynthesis in O. vulgaris, we have recently conducted a series of studies aiming to identify and characterise the function of genes encoding enzymes that mediate the conversions in the PUFA biosynthetic pathways. First, we identified a fatty acyl desaturase (Fad) cDNA sequence with homology to the vertebrate Fads family [6], enzymes that participate in long-chain $\left(\mathrm{C}_{20-24}\right)$ PUFA (LC-PUFA) biosynthetic pathways [7-9]. The expression of the common octopus Fad in yeast demonstrated that the enzyme was a $\Delta 5$ desaturase ( $\Delta 5 \mathrm{Fad}$ ) with high efficiency towards both saturated and polyunsaturated fatty acid (FA) substrates [6]. Thus, the $O$. vulgaris $\Delta 5 \mathrm{Fad}$ was able to desaturate the yeast endogenous saturated FAs 16:0 and 18:0 to the corresponding monoenes 16:1n-11 $\left({ }^{\Delta 5} 16: 1\right)$ and 18:1n-13 $\left({ }^{\Delta 5} 18: 1\right)$, respectively. Furthermore, the O. vulgaris $\Delta 5$ Fad efficiently desaturated the PUFA 20:4n-3 and 20:3n-6 to the $\Delta 5$ desaturation products eicosapentaenoic acid (EPA, 20:5n-3) and arachidonic acid (ARA, 20:4n-6), respectively (Figure 1).

A second study provided further evidence of the existence of an active PUFA biosynthetic system in the common octopus [10]. Thus, a cDNA encoding a protein with high homology to an elongation of very long-chain fatty acids (Elovl) protein was isolated [10]. Phylogenetic analysis comparing the amino acid (aa) sequence of the O. vulgaris Elovl with other elongases from molluscs and vertebrates clearly showed that the common octopus Elovl, as well as other putative elongases from molluscs, was grouped as a basal cluster of the vertebrate Elovl2 and Elovl5 families [10]. Consequently, such an elongase has been termed "Elov15/2" [11] or "Elovl2/5" [12,13]. Regarding its function, the common octopus Elovl2/5 exhibited substrate specificities resembling those of vertebrate Elovl5 but not Elovl2, as it efficiently elongated $C_{18-20}$ PUFAs [10] but had no activity towards $C_{22}$ substrates. This was hypothesised as one of the reasons accounting for the inability of cephalopods to biosynthesise docosahexaenoic acid (DHA; 22:6n-3) (Figure 1) [10,11] through the so-called "Sprecher pathway" requiring the production of 24:5n-3 as an intermediate in the pathway [14]. Among alternative Elovl-like enzymes with a role in the biosynthesis of LC-PUFAs such as DHA in the common octopus, the Elov14 elongase is an interesting candidate, as studies on teleost orthologues have shown that Elovl4 can efficiently catalyse the elongation of 22:5n-3 to 24:5n-3 [15-19], and recent studies have further demonstrated a similar elongation ability in molluscs [12].

The above studies on O. vulgaris [6,10], as well as those on homologous genes from the common cuttlefish Sepia officinalis [20], have enabled us to predict the biosynthetic pathways of PUFAs in cephalopods (Figure 1). Beyond the biosynthesis of standard PUFAs, i.e., FAs whose double bonds are always separated by a methylene group $\left(-\mathrm{CH}_{2}-\right)$ [9], one can predict that some pathways involving the $\Delta 5 \mathrm{Fad}$ and Elovl2/5 lead to the production of so-called "non-methylene-interrupted FAs" (NMI FAs), a particular type of PUFA that had been previously reported in other molluscan classes (bivalves, gastropods), as well in sponges, echinoderms and other phyla [21-23]. Analyses performed in wild-caught specimens of $O$. vulgaris confirmed that the polar lipid fractions of nephridium, male gonad, eye and caecum contained NMI FAs identified as $\Delta 5,1120: 2, \Delta 7,1320: 2, \Delta 5,11,1420: 3$ and $\Delta 7,1322: 2$ [10]. From the unsaturation pattern of these compounds, it became clear that, in addition to $\Delta 5 \mathrm{Fad}$, a further desaturase with $\Delta 9$ activity was likely involved in the NMI FA biosynthetic pathways accounting for the $\Delta 5,9$ unsaturation patterns typically found among these compounds [22,23]. The stearoyl-CoA desaturase (Scd), an enzyme that is expressed in virtually all living organisms [24], has $\Delta 9$ desaturation capability and thus appears to play a role in NMI FA biosynthesis [7].

Our overall aim is to characterise the biosynthetic pathways of PUFAs including NMI FAs in cephalopods. Using the common octopus O. vulgaris as model species, we herein isolated two cDNAs, namely Scd and Elovl4 sequences, and characterised their functions by heterologous expression in 
yeast. In order to establish the mechanisms accounting for biosynthesis of $\Delta 5,9$ dienes (NMI FA) we further investigated the roles that the herein characterised Scd and the previously reported $\Delta 5 \mathrm{Fad}$ [6] play within these pathways.

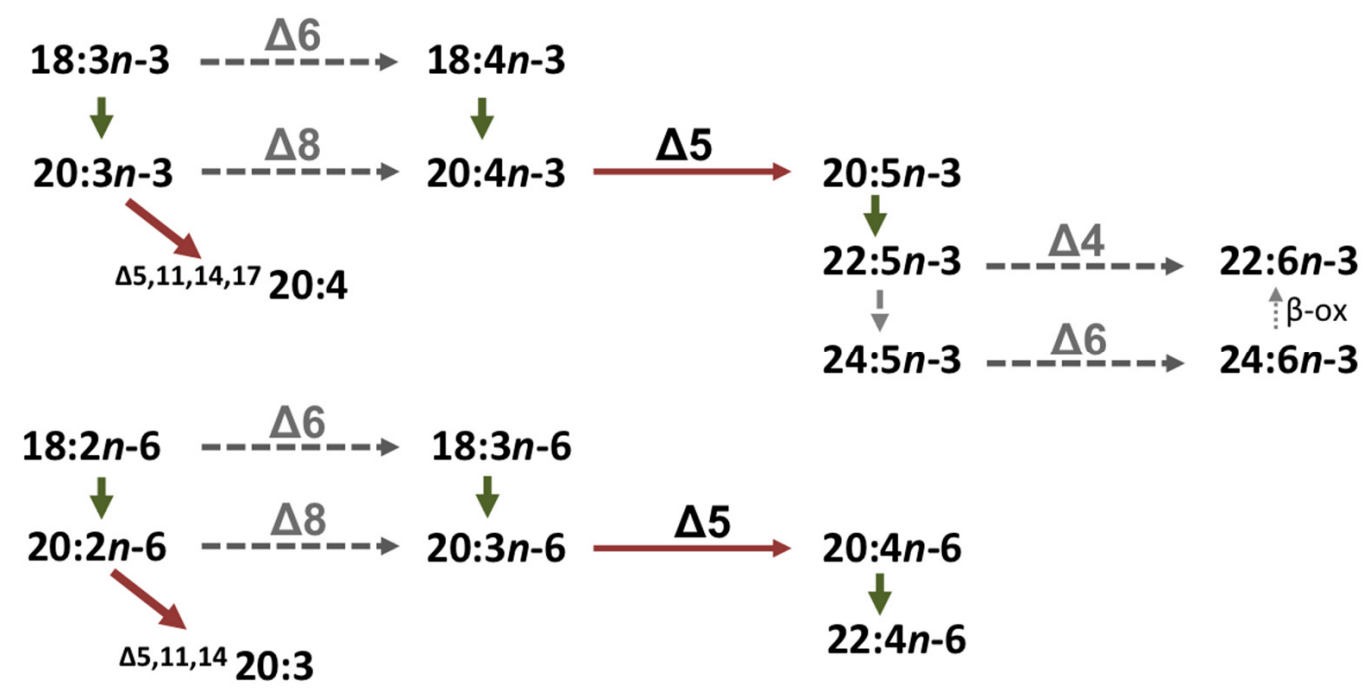

Figure 1. Model of biosynthetic pathways of polyunsaturated fatty acids in cephalopods. Enzymatic activities shown in the diagram are predicted from heterologous expression in yeast (Saccharomyces cerevisiae) of fatty acyl desaturases (red arrows) and elongation of very long-chain fatty acid (Elovl) proteins (green arrows) from Octopus vulgaris [6,10] and Sepia officinalis [20]. Dotted arrows indicate reactions that have not yet been demonstrated prior to the present study. $\beta$-ox, partial $\beta$-oxidation.

\section{Results}

\subsection{Octopus vulgaris Scd Sequence}

The O. vulgaris Scd-like cDNA consisted of a 981-bp open reading frame (ORF) encoding a putative protein of 326 amino acids (aa) with a predicted molecular weight of $37.9 \mathrm{kDa}$. Its sequence was deposited in the GenBank database with the accession number JX310655. In common with other Scd proteins, the O. vulgaris putative Scd possessed three histidine boxes (HXXXH, HXXHH and HXXHH), four membrane-spanning regions rich in hydrophobic aa, and lacked the cytochrome b5 domain characteristic of Fads (Figure 2).

The deduced aa sequence from the common octopus Scd was $50.8 \%-53.4 \%$ identical to Scd sequences from vertebrates including Homo sapiens (NP_005054.3), Gallus gallus (NP_990221.1) and Xenopus laevis (NP_001087809.1), and 40.7\% and 43.9\% identical to Scd from the nematode Caenorhabditis elegans FAT-5 (NP_507482.1) and FAT-6 (NP_001255595.1), respectively. When compared to mollusc Scd-like protein sequences, the O. vulgaris Scd showed relatively high identity scores with orthologues from Octopus bimaculoides (99.0\%) (XP_014788510.1), Crassostrea gigas (63.0\%) (XP_011452904.1) and Lottia gigantea (60.7\%). Importantly, identities between the newly cloned Scd and several Fads desaturases including the $\Delta 5$-like desaturase identified in O. vulgaris [6] were below $16.0 \%$. 


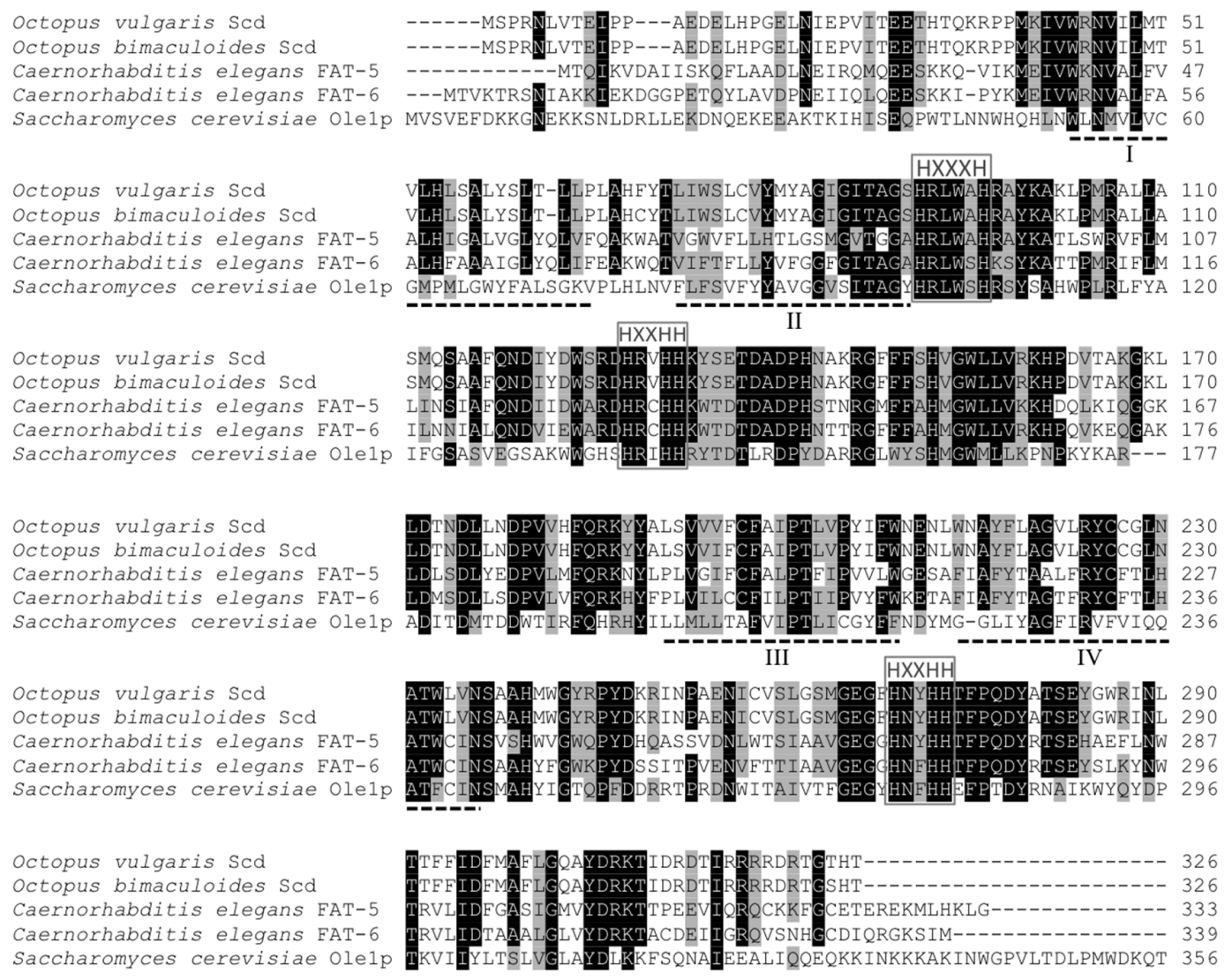

Figure 2. ClustalW amino acid alignment comparing the Octopus vulgaris stearoyl-CoA desaturase (Scd) with homologous sequences from the Octopus bimaculoides (XP_014788514.1), the Caenorabditis elegans FAT-5 (NP_507482.1) and the C. elegans FAT-6 (NP_001255595.1), and a portion of the Saccharomyces cerevisiae Ole1p sequence. Identical residues are shaded black and similar residues (using ClustalW2 default parameters) are shaded grey. The three histidine boxes (HXXXH, HXXHH and QXXHH) are highlighted with grey squares. Four (I-IV) trans-membrane domains predicted by Watts and Browse [25] are underlined with dashed lines.

\subsection{Octopus Elovl4-Like Sequence}

The Elovl4-like cDNA consisted of an ORF of $930 \mathrm{bp}$ whose deduced protein had 309 aa with a predicted molecular weight of $37.9 \mathrm{kDa}$ (deposited in GenBank database with accession number KJ590963). From analogy to vertebrate orthologues [15-18], five putative transmembrane domains containing hydrophobic aa stretches can be predicted (Figure 3). The common octopus putative Elovl4 contained the histidine dideoxy-binding motif $\mathrm{HXXHH}$, and the putative endoplasmic reticulum $(\mathrm{ER})$ retrieval signal with a histidine $(\mathrm{H})$ and lysine $(\mathrm{K})$ residues at the carboxyl terminus, HXKXX (Figure 3) [26].

Comparison of the deduced aa sequence of the O. vulgaris Elovl4 with other orthologues revealed high identity with the O. bimaculoides Elovl4 (93.5\%) (XP_014784234.1), with remarkable lower identity scores obtained when compared with Elovl4 sequences from L. gigantea (57.9\%) (XP_009051096.1), C. gigas (41.6\%) (XP_011450778.1), the sea squirt Ciona intestinalis (48.9\%) (AAV67802.1) [27], H. sapiens (50.8\%) (NP_073563.1), G. gallus (49.1\%) (NP_001184238.1) and Anolis carolinensis (47.6\%) (XP_003215742.1). Identity scores of $36.6 \%$ and $36.2 \%$ were obtained by comparing the O. vulgaris Elovl4 with the previously characterised Elovl2/5 from O. vulgaris (AFM93779.1) [10] and S. officinalis (AKE92956.1) [20], respectively. 


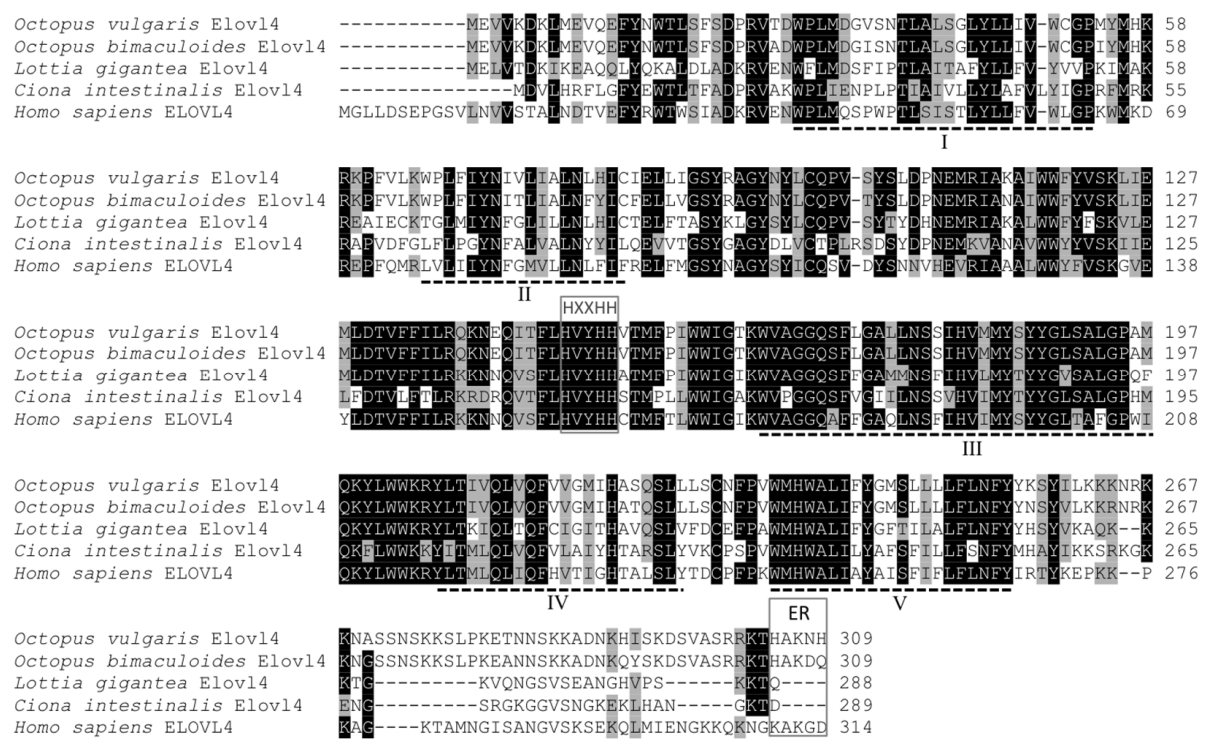

Figure 3. ClustalW amino acid alignment of the Octopus vulgaris Elovl4 with homologous sequences from Octopus bimaculoides (XP_014784234.1), Lottia gigantea (XP_009051096.1), Ciona intestinalis (AAV67802.1) and Homo sapiens (NP_073563.1). Identical residues are shaded black and similar residues (using ClustalW default parameters) are shaded grey. Indicated are the conserved histidine box motif $\mathrm{HXXHH}$, five (I-V) putative membrane-spanning domains, and the putative endoplasmic reticulum (ER) retrieval signal.

\subsection{Functional Characterisation of the Octopus Scd}

Yeast Saccharomyces cerevisiae cells lacking the OLE1 gene are unable to synthesise $\Delta 9$-monounsaturated FAs including palmitoleic acid (16:1n-7) and oleic acid (18:1n-9), which are essential for growth [28]. To address whether the O. vulgaris Scd was able to complement yeast OLE1 function, an S. cerevisiae ole1 $\Delta$ mutant strain (L8-14C) was transformed with a plasmid expressing the octopus Scd (p416OLE1-Scd) under the control of yeast OLE1 promoter. The ole1 $\Delta$ mutant yeast were also transformed with a plasmid expressing the S. cerevisiae OLE1 ( $\triangle 9$ desaturase) under the control of its own promoter (p416OLE1-OLE1) as a positive control, and with empty vector (pRS416) as a negative control. All three yeast transformants were able to grow in media supplemented with at least one supplemented FA (16:1n-7 and/or 18:1n-9) (Figure 4a-c). However, only yeast cells expressing either the S. cerevisiae OLE1 or the O. vulgaris Scd grew on medium that was not supplemented with monounsaturated FAs (Figure 4d). These results indicated that the common octopus Scd complemented the function of the yeast OLE1 desaturase.

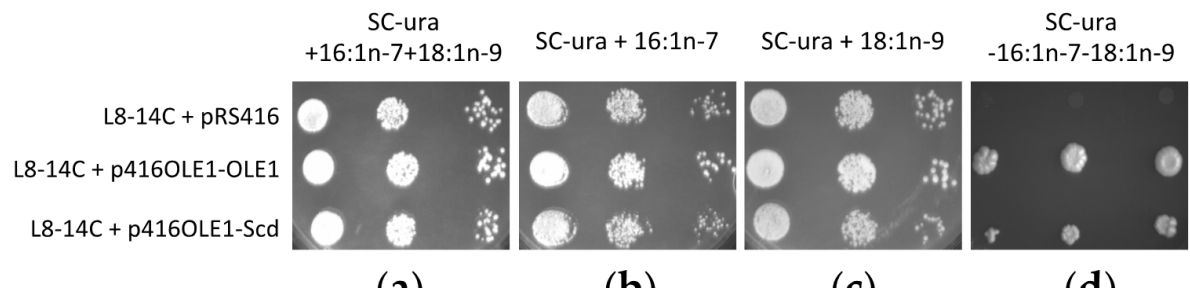

(a)

(b)

(c)

(d)

Figure 4. Complementation of the Saccharomyces cerevisiae ole1s mutant strain L8-14C with the Octopus vulgaris stearoyl-CoA desaturase (Scd) coding region. Yeast were transformed with the pRS416 empty vector (negative control), p416OLE1-OLE1 (expressing the S. cerevisiae OLE1 under the control of its own promoter) and p416OLE1-Scd (expressing the O. vulgaris Scd under the control of S. cerevisiae OLE1 promoter) and grown in SC medium lacking uracil (SC-ura) supplemented with 16:1n-7 and $18: 1 n-9(\mathbf{a}), 16: 1 n-7$ (b) or 18:1n-9 (c), or in the absence of these fatty acids (d). 
To analyse in more detail the complemention of yeast ole1 $\Delta$ mutants by the O. vulgaris Scd, we determined the FA composition of ole1 $\Delta$ yeast cells transformed with either p416OLE1-OLE1 (i.e., expressing the S. cerevisiae OLE1) or p416OLE1-Scd (i.e., expressing the O. vulgaris Scd), and grown in liquid medium with no exogenously supplemented FAs. Yeast expressing the O. vulgaris Scd grew notably slower compared to controls and formed clumps as previously described [29]. Moreover, the p416OLE1-Scd yeast contained significantly $(p \leq 0.05)$ less $16: 1 n-7$ and more $16: 0$ than control yeast expressing the S. cerevisiae OLE1, suggesting a low affinity of the O. vulgaris Scd towards 16:0. On the contrary, contents of 18:1n-9 showed no statistical differences between yeast cells expressing Scd or OLE1, indicative of the octopus Scd participating in the biosynthesis of 18:1n-9 similar to S. cerevisiae Ole1p. The distinctive substrate specificities of the O. vulgaris Scd were further emphasised by the higher 18:1n-9/16:1n-7 ratio of yeast complemented with the O. vulgaris Scd compared to that of OLE1 control yeast (Table 1).

The substrate specificities of the $O$. vulgaris Scd were further investigated through an overexpression assay using the S. cerevisiae strain InvSc1 (Invitrogen, Paisley, UK), possessing the endogenous Ole1p. Comparison of the FA profiles between the control yeast (i.e., transformed with the empty pYES2) and yeast expressing the O. vulgaris Scd (i.e., transformed with pYES2-Scd) confirmed a role of the common octopus Scd in the biosynthesis of monounsaturated FAs (Table 2). Thus, significant $(p \leq 0.05)$ increases in the contents of 18:1n-9 (oleic acid) in pYES2-Scd yeast compared to control yeast were observed. Parallel decreased levels of the saturated FA precursor 18:0 were detected in pYES2-Scd yeast $(p \leq 0.05)$. Additionally, other monoenes corresponding to 20:1n-11 and 22:1n-13 were detected in total lipids of InvSc1 yeast expressing the O. vulgaris Scd. These results confirmed that the $O$. vulgaris Scd is a $\Delta 9$ desaturase with activity towards saturated FAs with chain-lengths $\geq C_{18}$. In contrast, shorter FAs $\left(\leq \mathrm{C}_{16}\right)$ did not appear to be adequate substrates for the octopus Scd and, for instance, the content of 16:1n-7 in yeast expressing the common octopus Scd was significantly lower than that of control yeast $(p \leq 0.05)$ (Table 2).

Table 1. Fatty acid (FA) composition of the S. cerevisiae strain L8-14C transformed with the yeast-endogenous OLE1 (SC OLE1) or stearoyl-CoA desaturase from O. vulgaris (OV Scd). Results are expressed as an area percentage of total fatty acids (FAs) found in transformed yeast. Different letters for each FA or ratio indicate significant differences among treatments ( $t$-test, $p \leq 0.05)$.

\begin{tabular}{ccc}
\hline Fatty Acid & SC OLE1 & OV Scd \\
\hline $16: 0$ & $23.5 \pm 3.6^{\mathrm{b}}$ & $59.3 \pm 4.9^{\mathrm{a}}$ \\
$16: 1 n-9$ & $0.5 \pm 0.4^{\mathrm{a}}$ & $0.7 \pm 0.7$ \\
$16: 1 n-7$ & $35.0 \pm 2.2^{\mathrm{a}}$ & $4.5 \pm 1.0^{\mathrm{b}}$ \\
$18: 0$ & $8.0 \pm 1.7$ & $5.6 \pm 1.3$ \\
$18: 1 n-9$ & $31.8 \pm 2.5$ & $29.8 \pm 6.0$ \\
$18: 1 n-7$ & $1.1 \pm 0.2^{\mathrm{a}}$ & $0.0 \pm 0.0^{\mathrm{b}}$ \\
$18: 1 n-9 / 16: 1 n-7$ & $0.9 \pm 0.1^{\mathrm{b}}$ & $6.8 \pm 2.0^{\mathrm{a}}$ \\
\hline
\end{tabular}

Table 2. Fatty acid (FA) composition of the S. cerevisiae InvSc1 transformed with either empty pYES2 vector (Control) or the common octopus Scd open reading frame (ORF). Different letters for each FA indicate significant differences among treatments $(t$-test, $p \leq 0.05)$.

\begin{tabular}{ccc}
\hline Fatty Acid & Control & OV Scd \\
\hline $14: 0$ & $1.6 \pm 0.2^{\mathrm{a}}$ & $1.0 \pm 0.1^{\mathrm{b}}$ \\
$14: 1 n-5$ & $0.5 \pm 0.1$ & $0.7 \pm 0.1$ \\
$16: 0$ & $25.9 \pm 0.3^{\mathrm{a}}$ & $22.2 \pm 1.2^{\mathrm{b}}$ \\
$16: 1 n-7$ & $37.7 \pm 0.8^{\mathrm{a}}$ & $28.6 \pm 3.3^{\mathrm{b}}$ \\
$18: 0$ & $8.5 \pm 0.3^{\mathrm{a}}$ & $4.4 \pm 0.9^{\mathrm{b}}$ \\
$18: 1 n-9$ & $24.1 \pm 0.7^{\mathrm{b}}$ & $40.9 \pm 3.6^{\mathrm{a}}$ \\
$18: 1 n-7$ & $1.1 \pm 0.1$ & $1.1 \pm 0.2$ \\
$20: 0$ & $0.1 \pm 0.0$ & $0.1 \pm 0.0$ \\
$20: 1 n-11$ & N.D. ${ }^{\mathrm{b}}$ & $0.4 \pm 0.2^{\mathrm{a}}$ \\
$22: 0$ & $0.1 \pm 0.0$ & $0.1 \pm 0.0$ \\
$22: 1 n-13$ & N.D. ${ }^{\mathrm{b}}$ & $0.1 \pm 0.0^{\mathrm{a}}$ \\
\hline
\end{tabular}


In order to establish the biosynthetic pathways of NMI FAs with $\Delta 5,9$ unsaturation patterns (or their derivatives such as $\Delta 5,11$ ) found in the common octopus lipids [10], InvSc1 yeast transformed with pYES2-Scd were grown in the presence of the monoene 20:1n-15 $\left({ }^{\Delta 5} 20: 1\right)$, while InvSc1 yeast expressing the $\Delta 5$ Fad (i.e., transformed with pYES2-Fad) [6] were grown in the presence of 20:1n-9 $\left({ }^{\Delta 11} 20: 1\right)$ (Figure 5). Our results showed that the O. vulgaris Scd was unable to desaturate the substrate ${ }^{\Delta 5} 20: 1\left(20: 1 n\right.$-15) to ${ }^{\Delta 5,9} 20: 2$ (Figure 5a), although yeast transformed with pYES2-Fad were able to produce ${ }^{\Delta 5,11} 20: 2\left(2.7 \% \pm 0.2 \%\right.$ conversion), thus confirming activity as $\Delta 5$ desaturase on $\left.{ }^{\Delta 11} 20: 1\right)$ (Figure 5b).

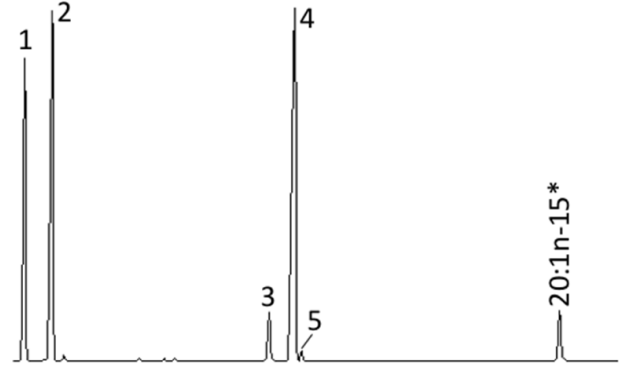

(a)

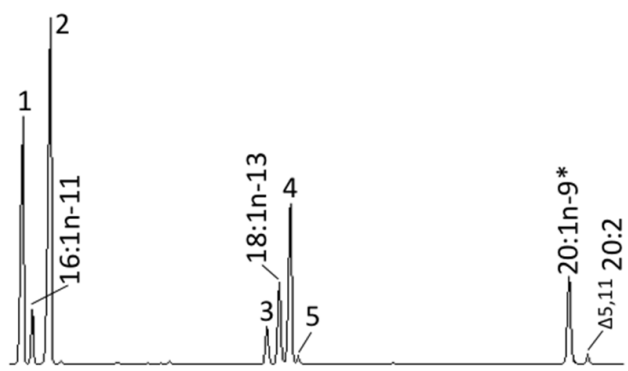

(b)

Figure 5. Biosynthesis of non-methylene interrupted fatty acids (FAs) in cephalopods: (a) Gas chromatography (GC) trace of yeast Saccharomyces cerevisiae expressing the Octopus vulgaris Scd and grown in the presence of 20:1n-15 ( $\left.{ }^{\Delta 5} 20: 1\right)$; (b) GC trace of yeast $S$. cerevisiae expressing the previously characterised O. vulgaris $\Delta 5$ Fad [6] and grown in the presence of 20:1n-9 $\left({ }^{\Delta 11} 20: 1\right)$. Peaks 1-5 represent S. cerevisiae endogenous FAs, namely 16:0 (1), 16:1 isomers (2), 18:0 (3), 18:1n-9 (4) and 18:1n-7 (5). Peaks derived from exogenously added substrates $\left({ }^{*}\right)$ and the desaturation product ${ }^{45,11} 20: 2(\mathbf{b})$ are indicated accordingly.

\subsection{Functional Characterisation of the Octopus Elovl4}

The role of the O. vulgaris Elovl4 in the biosynthesis of very long-chain $\left(>\mathrm{C}_{24}\right)$ PUFAs (VLC-PUFAs) was investigated in yeast $S$. cerevisiae (strain InvSc1) expressing the Elovl4 and grown in the presence of one of either $C_{18}\left(18: 3 n-3,18: 2 n-6,18: 4 n-3\right.$ and 18:3n-6), $C_{20}$ (20:5n-3 and 20:4n-6), $C_{22}(22: 5 n-3,22: 4 n-6$, 22:6n-3) or $\mathrm{C}_{24}$ (24:5n-3) PUFA substrates (Table 3). Gas Chromatography-Mass Spectrometry (GC-MS) analyses confirmed that the control yeast did not have the ability to elongate PUFAs, consistent with the previously reported lack of a PUFA elongase in S. cerevisiae strain InvSc1 [30]. However, the common octopus Elovl4 conferred the yeast the ability to elongate PUFAs to the corresponding elongated polyenoic products of both $n-3$ and $n-6$ series (Table 3). With the exception of DHA (22:6n-3), the addition of $C_{22}$ and $C_{24}$ PUFA substrates resulted in the production of polyenes of $C_{32}$ products or even $C_{34}$ when 24:5n-3 was used as substrate (Table 3). Indeed, the endogenous production of PUFAs with chain lengths $\geq C_{26}$ in yeast supplemented with exogenously supplemented $C_{22}$ and $C_{24}$ PUFAs allowed us to estimate the Elovl4 efficiency (as \% conversion) towards potential VLC-PUFA substrates that are not commercially available. The results showed that the highest $\%$ conversions (often over $88.0 \%$ ) were consistently detected on $C_{28}, C_{30}$ and $C_{32}$ substrates (Table 3 ). It is noteworthy that the octopus Elovl4 was able to convert the exogenously added 20:5n-3 and 22:5n-3 to 24:5n-3, the substrate for DHA biosynthesis via the Sprecher pathway [14], although it showed relative low elongase activity towards DHA itself, which was marginally elongated (0.7\%) to $24: 6 n-3$. 
Table 3. Role of the Octopus vulgaris Elovl4 in the biosynthesis of very long-chain $\left(>\mathrm{C}_{24}\right)$ polyunsaturated fatty acids (FAs). Conversions were calculated for each stepwise elongation according to the formula (areas of first product and longer chain products/(areas of all products with longer chain than substrate + substrate area) $) \times 100$. The substrate FA varies as indicated in each step-wise elongation.

\begin{tabular}{|c|c|c|c|}
\hline FA Substrate & Product & $\%$ Conversion & Elongation \\
\hline \multirow{2}{*}{$18: 3 n-3$} & $20: 3 n-3$ & 2.8 & $\mathrm{C} 18 \rightarrow 22$ \\
\hline & $22: 3 n-3$ & 3.0 & $\mathrm{C} 20 \rightarrow 22$ \\
\hline \multirow[b]{2}{*}{$18: 2 n-6$} & $20: 2 n-6$ & 1.1 & $\mathrm{C} 18 \rightarrow 22$ \\
\hline & $22: 2 n-6$ & 26.2 & $\mathrm{C} 20 \rightarrow 22$ \\
\hline $18: 4 n-3$ & $20: 4 n-3$ & 1.0 & $\mathrm{C} 18 \rightarrow 20$ \\
\hline $18: 3 n-6$ & $20: 3 n-6$ & 0.8 & $\mathrm{C} 18 \rightarrow 20$ \\
\hline \multirow{2}{*}{$20: 5 n-3$} & $22: 5 n-3$ & 1.9 & $\mathrm{C} 20 \rightarrow 24$ \\
\hline & $24: 5 n-3$ & 8.1 & $\mathrm{C} 22 \rightarrow 24$ \\
\hline \multirow{2}{*}{$20: 4 n-6$} & $22: 4 n-6$ & 1.0 & $\mathrm{C} 20 \rightarrow 24$ \\
\hline & $24: 4 n-6$ & 6.2 & $\mathrm{C} 22 \rightarrow 24$ \\
\hline \multirow{5}{*}{$22: 5 n-3$} & $24: 5 n-3$ & 4.8 & $\mathrm{C} 22 \rightarrow 32$ \\
\hline & $26: 5 n-3$ & 37.9 & $\mathrm{C} 24 \rightarrow 32$ \\
\hline & $28: 5 n-3$ & 95.0 & $\mathrm{C} 26 \rightarrow 32$ \\
\hline & $30: 5 n-3$ & 93.5 & $\mathrm{C} 28 \rightarrow 32$ \\
\hline & $32: 5 n-3$ & 51.9 & $\mathrm{C} 30 \rightarrow 32$ \\
\hline \multirow{5}{*}{$22: 4 n-6$} & $24: 4 n-6$ & 3.6 & $\mathrm{C} 22 \rightarrow 32$ \\
\hline & $26: 4 n-6$ & 44.5 & $\mathrm{C} 24 \rightarrow 32$ \\
\hline & $28: 4 n-6$ & 94.1 & $\mathrm{C} 26 \rightarrow 32$ \\
\hline & $30: 4 n-6$ & 91.5 & $\mathrm{C} 28 \rightarrow 32$ \\
\hline & $32: 4 n-6$ & 46.3 & $\mathrm{C} 30 \rightarrow 32$ \\
\hline $22: 6 n-3$ & $24: 6 n-3$ & 0.7 & $\mathrm{C} 22 \rightarrow 24$ \\
\hline \multirow{5}{*}{$24: 5 n-3$} & $26: 5 n-3$ & 1.4 & $\mathrm{C} 24 \rightarrow 34$ \\
\hline & $28: 5 n-3$ & 88.8 & $\mathrm{C} 26 \rightarrow 34$ \\
\hline & $30: 5 n-3$ & 88.6 & $\mathrm{C} 28 \rightarrow 34$ \\
\hline & $32: 5 n-3$ & 63.2 & $\mathrm{C} 30 \rightarrow 34$ \\
\hline & $34: 5 n-3$ & 17.3 & $\mathrm{C} 32 \rightarrow 34$ \\
\hline
\end{tabular}

\section{Discussion}

Fish and seafood are the primary sources of omega-3 long-chain $\left(\mathrm{C}_{20-24}\right)$ PUFAs for humans [31] and this partly explains the considerable interest in elucidating the PUFA biosynthetic pathways in aquatic and marine species, particularly in farmed fish for which current trends in feed formulation are impacting the nutritional quality for human consumers [32]. Previous investigations on O. vulgaris $[6,10,33]$ and S. officinalis [20] revealed that cephalopods possess active desaturase and elongase enzymes involved in the biosynthesis of PUFAs including NMI FAs. Using the common octopus $O$. vulgaris as model species, the present study aimed to expand our knowledge of roles that further desaturases and elongases could have on PUFA biosynthesis in cephalopods. Consequently, we characterised a stearoyl-CoA desaturase (Scd) with a putative role in the biosynthesis of NMI FAs, and an Elovl4 elongase that, in addition to its participation in the biosynthesis of VLC-PUFAs [15-19,34], could potentially catalyse the elongation of 22:5n-3 to 24:5n-3 required for DHA biosynthesis through the Sprecher pathway [14].

The Scd, present in virtually all living organisms [24], is an enzyme with $\Delta 9$ desaturase activity. Consistently, the newly-cloned O. vulgaris Scd cDNA was confirmed to encode a $\Delta 9$ desaturase that was able to operate on a range of saturated FA substrates with different chain lengths, particularly $\geq C_{18}$. These functions are similar to those described for two homologous sequences found in the nematode 
C. elegans, namely FAT-6 and FAT-7, which can efficiently desaturate 18:0 to 18:1n-9 but have lower activity towards 16:0 [25]. Interestingly, FAT-5, another Scd-like sequence existing in C. elegans, efficiently desaturated 16:0 to $16: 1 n-7$ but had nearly undetectable activity on $18: 0$, and it is thus regarded as a palmitoyl-CoA-specific desaturase [25]. The functional characterisation of the O. vulgaris Scd and its particularly high substrate affinity towards 18:0 is consistent with 18:1n-9 typically appearing several folds above the levels of shorter monoenes such as 16:1n-7 in lipids across the Mollusca phylum [21]. Beyond its role in biosynthesis of monounsaturated FA, occurrence of NMI FAs with $\Delta 5,9$ unsaturation patterns in lipids from molluscs [22] suggested a role of Scd in the biosynthesis of this particular type of PUFA. Our results revealed that the O. vulgaris Scd was not able to introduce a second double bond on a $\Delta 5$ monoene such as 20:1n-15 $\left({ }^{\Delta 5} 20: 1\right)$. While the possibility that cephalopod Scd can introduce $\Delta 9$ desaturations on other monoenes cannot be ruled out, these results strongly suggest that the role of Scd in the biosynthesis of NMI FAs with $\Delta 5,9$ unsaturation patterns is limited to the insertion of the first unsaturation at $\Delta 9$ position on saturated FAs. Indeed, the O. vulgaris $\Delta 5$ Fad, previously characterised as $\Delta 5$ desaturase [6], was able to introduce a $\Delta 5$ unsaturation on 20:1n-9 $\left({ }^{\Delta 11}\right.$ 20:1) producing the NMI FA ${ }^{\Delta 5,11} 20: 2$. Overall, these results allow us to hypothesise that the insertion of double bonds required for NMI FA biosynthesis is initiated with a $\Delta 9$ desaturation by Scd and a subsequent $\Delta 5$ desaturation catalysed by $\Delta 5$ Fad. Compared to other molluscan classes, the occurrence of NMI FAs in cephalopods appears to be rather limited and hence the contribution of endogenous production of NMI FAs vs. dietary input is difficult to establish. Nevertheless, the mechanism of ${ }^{\Delta 5,11} 20: 2$ biosynthesis postulated herein aligns well with the occurrence of ${ }^{\Delta 5,11} 20: 2$ in the polar lipids of nephridia of adult O. vulgaris [10], suggesting that NMI FA biosynthesis is possible in cephalopods and likely other molluscs.

In addition to desaturases, certain Elovl enzymes play crucial roles in the biosynthesis of essential PUFAs such as EPA, ARA and DHA $[9,11,26]$. Whereas the previously characterised cephalopod Elovl2/5 demonstrated an ability to efficiently elongate $C_{18}$ and $C_{20}$ PUFA substrates $[6,20]$, lack of elongase capability towards 22:5n-3 was hypothesised as a major limiting factor for DHA biosynthesis through the Sprecher pathway $[6,20]$. Functional characterisation of the O. vulgaris Elovl4 demonstrated the ability of this enzyme to contribute potentially to DHA biosynthesis by producing 24:5n-3, which is the substrate for $\Delta 6$ desaturation and chain-shortening to produce DHA in the Sprecher pathway [14]. Such an ability of Elovl4 has been reported in fish orthologues [15-19]. Despite the elongation capability of $O$. vulgaris herein revealed, our overall observations on gene repertoire and function $[6,10,20,33]$ strongly suggest that DHA is still an essential FA for cephalopods. The limited ability to biosynthesise DHA is related, rather than to an issue with elongase activity, to the apparent absence of key desaturation abilities ( $\Delta 6$ or $\Delta 4)$ required for DHA biosynthesis according to the two aerobic pathways known in vertebrates (Figure 1). In addition to the abovementioned $\Delta 6$ desaturation required in the Sprecher pathway [14], an alternative pathway described in some teleost fish involves a $\Delta 4$ desaturation from 22:5n-3 to produce DHA directly (Figure 1) [9]. Molecular evidence provided by the recently published Octopus bimaculoides genome project [35] suggests that cephalopods possess one single Fad-like desaturase in their genome and this is likely to be a $\Delta 5$ desaturase since all the Fad-like desaturase functionally characterised not only from cephalopods such as O. vulgaris and S. officinalis [6,10] but also from bivalves [36] and gastropods [37], have $\Delta 5$ desaturase activities. In non-cephalopod molluscs, further Fad-like desaturases exist [38] but, with the exception of the $\Delta 8$ desaturase found in the scallop Chlamys nobilis [39], their functions have not been determined and therefore it is yet not possible to predict whether $\Delta 6$ and/or $\Delta 4$ desaturase activities exist to enable DHA biosynthesis in those species.

The O. vulgaris Elovl4 is also involved in the biosynthesis of VLC-PUFAs as it was able to elongate exogenously supplemented PUFAs ranging from $C_{18-24}$ to PUFA products with chain lengths of $\geq C_{26}$. Such conversions are largely consistent with those previously exhibited by human [34] and fish [15-19] Elovl4 proteins. Surprisingly, no elongation products beyond $\mathrm{C}_{24}$ were reported in the functional characterisation of the bivalve C. nobilis Elovl4 [39]. The elongation activities of the O. vulgaris Elovl4 
estimated in the yeast expression system strongly suggested that this enzyme is particularly efficient towards $\mathrm{C}_{26-30}$ substrates, for which the highest \% conversions were observed. Similarly, the human ELOVL4 had PUFAs of $\geq \mathrm{C}_{26}$ as preferred substrates for elongation, although the human ELOVL4 was able to produce up to $\mathrm{C}_{38}$ PUFA elongation products in mammalian cell lines [34]. Due to technical challenges in their analysis and relatively low abundance, the functions of VLC-PUFAs are not fully understood [40]. Nevertheless, the structural features of VLC-PUFAs combining those from saturated FAs at one end and those from PUFAs at the other allow unique membrane lipid conformations in photoreceptors and spermatozoa, thus suggesting important roles of VLC-PUFAs in vision and reproduction of vertebrates [40-43]. Investigations of VLC-PUFAs in cephalopods and, therefore, the herein reported activities of the O. vulgaris Elovl4 cannot be correlated with the presence of VLC-PUFAs in vivo. Interestingly, some very long-chain NMI FAs have been reported in nudibranchs $[44,45]$ but, unfortunately, the potential role of Elovl4 in the biosynthesis of such compounds was not determined. Interestingly, unpublished data on tissue distribution analysis of the common octopus Elov14 mRNA indicated that gonads and, to a lesser extent eye, were also major sites for VLC-PUFA biosynthesis in cephalopods.

In conclusion, the present study demonstrated that the common octopus O. vulgaris possesses an Scd with high affinity for saturated FA substrates with chain lengths of 18 carbons or longer. Its inability to introduce a double bond into $\Delta 5$ monoene strongly suggested that the role of the Scd in NMI FA biosynthesis was restricted to the introduction of the first double bond at the $\Delta 9$ position, whereas the $O$. vulgaris $\Delta 5$ Fad can introduce the second unsaturation at the $\Delta 5$ position according to the herein confirmed ability to convert 20:1n-9 to $\Delta 5,1120: 2$, an NMI FA previously reported in nephridia of $O$. vulgaris adult specimens. Beyond desaturases, we could also demonstrate that O. vulgaris possesses an Elovl4 responsible for the biosynthesis of VLC-PUFAs.

\section{Materials and Methods}

\subsection{Tissue Samples}

Tissue samples including brain, nerve, muscle, heart, hepatopancreas, gill, caecum, eye, nephridium and gonads were obtained from the dissection of two (male and female) common octopus adult specimens as previously described [6,10]. Briefly, two (male and female) wild O. vulgaris adults $(\sim 1.5 \mathrm{~kg})$ were maintained in seawater tanks at the facilities of the Instituto de Acuicultura Torre de la Sal; before they were cold, they were anesthetised and sacrificed by direct brain puncture. After collection, samples were immediately frozen at $-80^{\circ} \mathrm{C}$ until further analysis. Total RNA was extracted from octopus tissues using TriReagent ${ }^{\circledR}$ (Sigma-Aldrich, Alcobendas, Spain) according to manufacturer's instructions. Two $\mu \mathrm{g}$ of total RNA tissue samples were used for synthesis of first strand cDNA using M-MLV reverse transcriptase (Promega, Southampton, UK) primed with random hexamers.

\subsection{Molecular Cloning of the Scd and Elovl4 cDNA Sequences}

The full-length sequences of the Scd and Elovl4 cDNA were obtained as follows. The deduced aa sequences of Scd proteins from Homo sapiens (NP_005054.3), Gallus gallus (NP_990221.1), Anolis carolinensis (XP_003226591.1), Caenorhabditis elegans (FAT-7) (NP_504814.1), Acheta domesticus (AAK25796.1) and Pediculus humanus (XP_002424386.1) were aligned using BioEdit v5.0.6 (Tom Hall, Department of Microbiology, North Carolina State University, Raleigh, NC, USA). Conserved regions were used for in silico searches of mollusc expressed sequence tags (EST) using The National Center for Biotechnology Information (NCBI) tblastn tool (http:/ / www.ncbi.nlm.nih.gov/). Processed Scd-like Expressed Sequence Tags (ESTs) from the molluscs Lottia gigantea (FC767047.1 and FC644199.1), Aplysia californica (FF074235.1, FF076278.1, EB307164.1, EB253812.1 and EB281044.1), Limnaea stagnalis (ES580199.1 and ES579822.1), Crassostrea gigas (CU997931.1 and FP006991.1), Euprymna scolopes (DW280836.1, DW269578.1 and DW273589.1) and Ruditapes decussatus (AM870139.1), were aligned 
(Bioedit) and conserved regions used for the design of the degenerate primers UNID9F ( $5^{\prime}$-ATCACAGCTGGWGCTCAYCG-3') and UNID9R (5'-TGGCATTGTGWGGGTCWGCATC-3').

To clone the first fragment of the octopus Elovl4, blastn searches of mollusc ESTs were performed using the so-called "transcript 2" from L. gigantea that was previously identified by [6] (gi |Lotgi1 |178149|) as query. Thus, additional Elovl4-like consensus sequences derived from ESTs from A. californica (EB285681.1, GD233360.1, EB316848.1, GD212825.1, EB325217.1, EB345626.1, EB345430.1), Saccostrea kagaki (AB375033.1) and C. gigas (HS215834.1, AM866458.1) were obtained and aligned with L. gigantea Elovl4-like to design the degenerate primers UNIE4F (5'-GCCAAGGCATTRTGGTGGTT-3') and UNIE4R (5'-GTSAGRTATCKYTTCCACCA-3').

Polymerase chain reactions (PCR) were performed with the GoTaq ${ }^{\circledR}$ Green Master Mix (Promega) and using a mixture of cDNAs from brain, nerve and hepatopancreas as template. The PCR consisted of an initial denaturing step at $95^{\circ} \mathrm{C}$ for $2 \mathrm{~min}$, followed by 35 cycles of denaturation at $95^{\circ} \mathrm{C}$ for $30 \mathrm{~s}$, annealing at $50{ }^{\circ} \mathrm{C}$ for $30 \mathrm{~s}$, extension at $72{ }^{\circ} \mathrm{C}$ for $40 \mathrm{~s}$, followed by a final extension at $72{ }^{\circ} \mathrm{C}$ for $5 \mathrm{~min}$. PCR products of approximately $180 \mathrm{bp}$ (Scd) and $290 \mathrm{bp}$ (Elovl4) were obtained and thereafter confirmed as positive by sequencing (DNA Sequencing Service, IBMCP-UPV, Valencia, Spain). Full-length cDNA of the octopus Scd and Elovl4 were completed by $5^{\prime}$ and $3^{\prime}$ rapid amplification of cDNA ends (RACE) PCR (FirstChoice RLM-RACE kit, Ambion, Applied Biosystems, Warrington, UK) with gene-specific primers shown in Table S1.

\subsection{Sequence Analysis}

The deduced aa sequences of the O. vulgaris Scd and Elovl4 ORF were compared to corresponding orthologues from other invertebrate and vertebrate species and sequence identity scores were calculated using the EMBOSS Needle Pairwise Sequence Alignment tool (http://www.ebi.ac.uk/ Tools/psa/emboss_needle/). Deduced aa sequence alignments were carried out using the built-in ClustalW tool (BioEdit v7.0.9, Tom Hall, Department of Microbiology, North Carolina State University, Raleigh, NC, USA).

\subsection{Functional Characterisation of Octopus Scd: Complementation Assay of the S. cerevisiae ole1 $\Delta$ Mutant Strain L8-14C}

The open reading frame (ORF) of the common octopus Scd was amplified from a mixture of cDNAs (brain, nerve and hepatopancreas) using the high fidelity $P f u$ Turbo DNA polymerase (Promega) and the primers OVD9VF and OVD9VR, containing restriction sites HindIII and XhoI, respectively (underlined in Table S1). PCR conditions consisted of an initial denaturing step at $95^{\circ} \mathrm{C}$ for $2 \mathrm{~min}$, followed by 32 cycles of denaturation at $95^{\circ} \mathrm{C}$ for $30 \mathrm{~s}$, annealing at $58^{\circ} \mathrm{C}$ for $30 \mathrm{~s}$, extension at $72{ }^{\circ} \mathrm{C}$ for $2.5 \mathrm{~min}$, followed by a final extension at $72^{\circ} \mathrm{C}$ for $5 \mathrm{~min}$. After restriction of the PCR product, the O. vulgaris Scd ORF was cloned into the yeast expression vector p416TEF (a centromeric plasmid with a URA3 selectable marker) to produce the construct p416TEF-Scd, in which octopus Scd was under the control of the yeast TEF1 promoter. Subsequently, the promoter region of the S. cerevisiae OLE1 $\triangle 9$-fatty acid desaturase gene amplified from yeast genomic DNA with the primers SCPromOLE1F and SCPromOLE1R containing SacI and HindIII sites, respectively, was cloned upstream of the O. vulgaris Scd, replacing the TEF1 promoter and producing the construct p416OLE1-Scd, in which octopus Scd is expressed under the control of the yeast OLE1 promoter. As a positive control, the promoter and ORF of $S$. cerevisiae OLE1 were amplified by PCR from yeast genomic DNA using the primers SCPromOLE1F and SCOLE1R that contained restriction sites for SacI and XhoI, respectively (Table S1). To obtain the p416OLE1-OLE1 plasmid, the OLE1 PCR fragment was cloned into p416TEF SacI and XhoI restriction sites, allowing TEF1 promoter replacement by OLE1 promoter and ORF.

To address the common octopus Scd function we used S. cerevisiae L8-14C (MATa ole1 $1:: L E U 2$, leu2-3,112, trp1-1, ura3-52, his4; kindly donated by Dr. Charles E. Martin), a strain whose OLE1 gene has been deleted. Therefore, L8-14C cells lack the only yeast $\Delta 9$-fatty acid desaturase and require the supply of monounsaturated FAs including $0.5 \mathrm{mM}$ palmitoleic $(16: 1 n-7)$ and $0.5 \mathrm{mM}$ oleic $(18: 1 n-9)$ acids in the 
medium for growth. To address whether the octopus Scd complemented the growth defect displayed by L8-14C cells in media lacking FAs, yeast mutants transformed with pRS416 (empty vector as negative control), p416OLE1-OLE1 (positive control) or p416OLE1-Scd plasmids were grown in liquid SC medium lacking uracil (SC-ura) with supplemented FAs until exponential phase, and then assayed for growth on plates in 10-fold serial dilution drops starting at $\mathrm{OD}_{600}=0.1$. Plates were incubated for 3 days at $30^{\circ} \mathrm{C}$ and photographed. To determine FA composition, four L8-14C colonies from yeast transformed with either p416OLE1-OLE1 or p416OLE1-Scd were grown in $10 \mathrm{~mL}$ of yeast extract peptone dextrose (YPD) broth lacking 18:1n-9 and 16:1n-7. After incubation at $30{ }^{\circ} \mathrm{C}$ for $48 \mathrm{~h}$, a $5 \mathrm{~mL}$ aliquot of yeast culture was pelleted (1000 g for $2 \mathrm{~min}$ ), washed twice with $\mathrm{dd}_{2} \mathrm{O}$, homogenised in chloroform/methanol $(2: 1, v / v)$ containing $0.01 \%$ butylated hydroxy toluene (BHT) [30,46], and kept at $-20{ }^{\circ} \mathrm{C}$ until further analysis.

\subsection{Functional Characterisation of Octopus Scd: Overexpression in the S. cerevisiae Strain InvSc1}

Primers containing HindIII (forward) and XhoI (reverse) restriction sites (underlined in Table S1) OVD9VF and OVD9VR (Scd) were used to amplify the ORF of the O. vulgaris Scd, using the high fidelity $P f u$ Turbo DNA polymerase (Promega). Further cloning into the yeast expression vector pYES2 (Invitrogen), which contains a GAL1 promoter that is inducible by galactose and has URA3 as selective marker, was achieved after ligation of restricted ORF amplicons and plasmid pYES2 to produce the construct PYES2-Scd. The recombinant plasmids pYES2-Scd or pYES2 empty (negative control) were transformed into S. cerevisiae competent cells InvSc1 (S.c. EasyComp Transformation Kit, Invitrogen). Yeast were grown in SC-ura for 3 days.

One single yeast colony containing the pYES2-Scd or pYES2 was grown overnight at $30{ }^{\circ} \mathrm{C}$ in $5 \mathrm{~mL}$ of liquid SC-ura. Cell cultures were then used to inoculate $10 \mathrm{~mL}$ of fresh SC-ura for a final $\mathrm{OD}_{600}$ of 0.4. Four replicates for each construct (pYES2-Scd or pYES2) were run. Cells were grown at $30{ }^{\circ} \mathrm{C}$ for $5 \mathrm{~h}$ before the expression of the transgene was induced by the addition of galactose to $2 \%(w / v)$ [46]. After $48 \mathrm{~h}$ of galactose induction, yeast samples were collected, washed and homogenised in chloroform/methanol $(2: 1, v / v)$ containing $0.01 \%$ BHT. Samples were kept at $-20{ }^{\circ} \mathrm{C}$ until further analysis.

\subsection{Role of the Octopus Scd and $\Delta 5$ Fad in the Biosynthesis of Non-Methylene-Interrupted FAs}

In order to establish the role of Scd and the previously characterised $\Delta 5 \mathrm{Fad}$ [6] in the biosynthetic pathways of $\Delta 5,9$ (or $\Delta 5,11$ ) non-methylene interrupted FAs (NMI FAs) we conducted the following experiment. First, yeast InvSc1 transformed with pYES2-Scd, i.e., expressing the octopus Scd, were grown in the presence of exogenously added 5-eicosenoic acid (20:1n-15 or ${ }^{\Delta 5}$ 20:1). Second, yeast InvSc1 transformed with pYES2-Fad, i.e., expressing the octopus Fads that was previously reported to exhibit $\Delta 5$-desaturase [6], were grown in the presence of 11-eicosenoic acid (20:1n-9 or $\left.{ }^{\Delta 11} 20: 1\right)$. Final concentration of exogenously-added substrates $\left({ }^{\Delta 5} 20: 1\right.$ or $\left.{ }^{\Delta 11} 20: 1\right)$ was $0.75 \mathrm{mM}$. Culture conditions and yeast sample collection were performed as described above. Yeast transformed with empty pYES2 were also grown in presence of ${ }^{\Delta 5} 20: 1$ and ${ }^{\Delta 11} 20: 1$ as control treatments.

\subsection{Functional Characterisation of Octopus Elovl4: Expression in the S. cerevisiae Strain InvSc1}

The octopus Elovl4 was functionally characterised by heterologous expression in yeast S. cerevisiae (strain InvSc1, Invitrogen). Similarly, as described above for the Scd overexpression experiment, the octopus Elovl4 ORF was amplified with primers OVE4VF and OVE4VR containing restriction sites for HindIII and XhoI, respectively (Table S1) which allowed its cloning into pYES2 to produce the construct pYES2-Elovl4. Yeast InvSc1 transformed with pYES2-Elovl4 were grown in SC-ura plates. One colony was subsequently grown in SC-ura broth to produce a bulk culture that allowed us to establish subcultures of $\mathrm{OD}_{600} 0.4$ in Erlenmeyer flasks that contained $5 \mathrm{~mL}$ of SC-ura broth and were in some cases supplemented with potential PUFA substrates for fatty acyl elongases. In order to assess the ability of the octopus Elovl4 to elongate PUFA substrates, yeast transformed with pYES2-Elovl4 
were grown in the presence of one of the following PUFA substrates: 18:3n-3, 18:2n-6, 18:4n-3, 18:3n-6, 20:5n-3, 20:4n-6, 22:5n-3, 22:4n-6, 22:6n-3 and 24:5n-3. The FA substrates were added to the yeast cultures at final concentrations of $0.5\left(\mathrm{C}_{18}\right), 0.75\left(\mathrm{C}_{20}\right), 1.0\left(\mathrm{C}_{22}\right)$ and $1.2\left(\mathrm{C}_{24}\right) \mathrm{mM}$ to compensate for decreased efficiency of uptake with increased chain length [47].

To test the ability of the octopus Elovl4 to elongate saturated FAs, yeast transformed with pYES2-Elov14 or pYES2 (negative control) were grown in the absence of exogenously added substrates and the capability of the Elovl4 to elongate saturated FAs was estimated by comparing the saturated FA profiles of both transformants. Three different replicates for each treatment were established.

\subsection{Fatty Acid Analysis by GC-MS}

Lipid extracts [48] from the transgenic yeast were utilised for preparing fatty acid methyl esters (FAME) as previously described [6,10]. Briefly, FAME were identified and quantified using an Agilent 6850 Gas Chromatograph coupled to a 5975 series Mass Selective Detector (MSD, Agilent Technologies, Santa Clara, CA, USA). The activity of the newly cloned octopus Scd was estimated by comparing the FA profiles (expressed as \% of total FAs) of control yeast with those from yeast transformed with p416OLE1-Scd (complementation experiment) or pYES2-Scd (overexpression experiment). The efficiency of the $O$. vulgaris $\Delta 5$ Fad to desaturate ${ }^{\Delta 11} 20: 1$ into the NMI FAs ${ }^{\Delta 5,11} 20: 2$ was calculated as \% conversion according to the formula: (area of product/(area of product + area of substrate)) $\times 100$. The double bond positions in FA products derived from conversion by transgenic yeast towards ${ }^{\Delta 5} 20: 1$ and ${ }^{\Delta 11} 20: 1$ was confirmed by preparing FA picolinyl ester derivatives according to the methodology described by [49] and modified according to [10]. Finally, the ability of the O. vulgaris Elovl4 to elongate the exogenously-added PUFA substrates (18:3n-3, 18:2n-6, 18:4n-3, 18:3n-6, 20:5n-3, 20:4n-6, 22:5n-3, 22:4n-6, 22:6n-3 and 24:5n-3) was calculated by the step-wise proportion of substrate FAs converted to elongated product as (areas of first product and longer chain products/(areas of all products with longer chain than substrate + substrate area) $) \times 100$ [50].

\subsection{Statistical Analyses}

For the functional characterisation experiments (complementation and overexpression) of the octopus Scd, FA analyses from yeast samples were expressed as mean values \pm standard deviation $(n=4)$. Similarly, the assay aiming to determine the ability of the octopus Elovl4 for elongation saturated FA was run in replicates $(n=3)$ and FA contents expressed as mean values \pm standard deviation. Homogeneity of variances was checked by Barlett's test. Comparison of FA profiles from control and yeast expressing the O. vulgaris Scd (complementation and overexpression experiments) or Elovl4 were compared with a Student's $t$-test. Comparisons of the means with $p$ values less or equal than 0.05 were considered significantly different. All the statistical analyses were carried out using the SPSS statistical package (SPSS Inc., Chicago, IL, USA).

\subsection{Materials}

All PUFA substrates were purchased from Nu-Chek Prep, Inc. (Elysian, MN, USA), except stearidonic acid (18:4n-3) from Sigma-Aldrich (Alcobendas, Spain) and tetracosapentaenoic acid (24:5n-3) from Larodan (Larodan Fine Chemicals AB, Malmö, Sweden). All chemicals used to prepare the S. cerevisiae media were from Sigma-Aldrich, except for the bacteriological agar obtained from Oxoid Ltd. (Hants, UK). 
Supplementary Materials: The following are available online at www.mdpi.com/1660-3397/15/3/82/s1, Table S1: Primer sequences used in the present study.

Acknowledgments: This research and OM were supported by a Marie Curie Reintegration Grant within the 7th European Community Framework Programme (PERG08-GA-2010-276916, LONGFA), with additional support from "Ministerio de Ciencia e Innovación" through the OCTOPHYS Project (AGL-2010-22120-C03-02) and a Juan de la Cierva postdoctoral contract to OM. Further support was obtained from the Generalitat Valenciana through grants within the PROMETEO (2010/006), "Grupos Emergentes" (GV/2013/123) programes, and AGL2011-29099 and BIO2014-56298-P grants from the Spanish Ministry of Economy and Competitiveness. R.D.L. was supported by a postdoctoral JAE-Doc contract from the Spanish Research Council (CSIC) and the European Social Fund.

Author Contributions: O.M., S.P. and J.C.N. conceived and designed the experiments; O.M., R.d.L. and F.H. performed the experiments; O.M., R.d.L. and I.V. analysed the data; I.V., S.P. and J.C.N. contributed reagents/materials/analysis tools; O.M., D.R.T. and J.C.N. wrote the paper.

Conflicts of Interest: The authors declare no conflict of interest. The founding sponsors had no role in the design of the study; in the collection, analyses, or interpretation of data; in the writing of the manuscript, and in the decision to publish the results.

\section{References}

1. Iglesias, J.; Fuentes, L.; Villanueva, R. Cephalopod Culture; Springer: Dordrecht, The Netherlands, $2014 ;$ p. 494.

2. Iglesias, J.; Sánchez, F.J.; Bersano, J.G.F.; Carrasco, J.F.; Dhont, J.; Fuentes, L.; Linares, F.; Muñoz, J.L.; Okumura, S.; Roo, J.; et al. Rearing of Octopus vulgaris paralarvae: Present status, bottlenecks and trends. Aquaculture 2007, 266, 1-15. [CrossRef]

3. Navarro, J.C.; Monroig, Ó.; Sykes, A.V. Nutrition as a key factor for cephalopod aquaculture. In Cephalopod Culture; Iglesias, J., Fuentes, L., Villanueva, R., Eds.; Springer: Dordrecht, The Netherlands, 2014; pp. 77-96.

4. Navarro, J.C.; Villanueva, R. Lipid and fatty acid composition of early stages of cephalopods: An approach to their lipid requirements. Aquaculture 2000, 183, 161-177. [CrossRef]

5. Navarro, J.C.; Villanueva, R. The fatty acid composition of Octopus vulgaris paralarvae reared with live and inert food: Deviation from their natural fatty acid profile. Aquaculture 2003, 219, 613-631. [CrossRef]

6. Monroig, Ó.; Navarro, J.C.; Dick, J.R.; Alemany, F.; Tocher, D.R. Identification of a $\Delta 5$-like fatty acyl desaturase from the cephalopod Octopus vulgaris (Cuvier 1797) involved in the biosynthesis of essential fatty acids. Mar. Biotechnol. 2012, 14, 411-422. [CrossRef] [PubMed]

7. Guillou, H.; Zadravec, D.; Martin, P.G.P.; Jacobsson, A. The key roles of elongases and desaturases in mammalian fatty acid metabolism: Insights from transgenic mice. Prog. Lipid Res. 2010, 49, 186-199. [CrossRef] [PubMed]

8. Monroig, Ó.; Navarro, J.C.; Tocher, D.R. Long-chain polyunsaturated fatty acids in fish: Recent advances on desaturases and elongases involved in their biosynthesis. In Proceedings of the XI International Symposium on Aquaculture Nutrition; Cruz-Suarez, L.E., Ricque-Marie, D., Tapia-Salazar, M., Nieto-López, M.G., Villarreal-Cavazos, D.A., Gamboa-Delgado, J., Hernández-Hernández, L.H., Eds.; Universidad Autónoma de Nuevo León: Monterrey, Mexico, 2011; pp. 257-282.

9. Castro, L.F.C.; Tocher, D.R.; Monroig, O. Long-chain polyunsaturated fatty acid biosynthesis in chordates: Insights into the evolution of Fads and Elovl gene repertoire. Prog. Lipid Res. 2016, 62, 25-40. [CrossRef] [PubMed]

10. Monroig, Ó.; Guinot, D.; Hontoria, F.; Tocher, D.R.; Navarro, J.C. Biosynthesis of essential fatty acids in Octopus vulgaris (Cuvier, 1797): Molecular cloning, functional characterisation and tissue distribution of a fatty acyl elongase. Aquaculture 2012, 360-361, 45-53. [CrossRef]

11. Monroig, Ó.; Tocher, D.R.; Navarro, J.C. Biosynthesis of polyunsaturated fatty acids in marine invertebrates: Recent advances in molecular mechanisms. Mar. Drugs 2013, 11, 3998-4018. [CrossRef] [PubMed]

12. Liu, H.; Zheng, H.; Wang, S.; Wang, Y.; Li, S.; Liu, W.; Zhang, G. Cloning and functional characterization of a polyunsaturated fatty acid elongase in a marine bivalve noble scallop Chlamys nobilis Reeve. Aquaculture 2013, 416, 146-151. [CrossRef]

13. Monroig, Ó.; Lopes-Marques, M.; Navarro, J.C.; Hontoria, F.; Ruivo, R.; Santos, M.M.; Venkatesh, B.; Tocher, D.R.; Castro, L.F.C. Evolutionary wiring of the polyunsaturated fatty acid biosynthetic pathway. Sci. Rep. 2016, 6, 20510. [CrossRef] [PubMed]

14. Sprecher, H. Metabolism of highly unsaturated n-3 and n-6 fatty acids. Biochim. Biophys. Acta 2000, 1486, 219-231. [CrossRef] 
15. Monroig, Ó.; Rotllant, J.; Cerdá-Reverter, J.M.; Dick, J.R.; Figueras, A.; Tocher, D.R. Expression and role of Elovl4 elongases in biosynthesis of very long-chain fatty acids during zebrafish Danio rerio early embryonic development. Biochim. Biophys. Acta 2010, 1801, 1145-1154. [CrossRef] [PubMed]

16. Monroig, Ó.; Webb, K.; Ibarra-Castro, L.; Holt, G.J.; Tocher, D.R. Biosynthesis of long-chain polyunsaturated fatty acids in marine fish: Characterization of an Elovl4-like elongase from cobia Rachycentron canadum and activation of the pathway during early life stages. Aquaculture 2011, 312, 145-153. [CrossRef]

17. Carmona-Antoñanzas, G.; Monroig, Ó.; Dick, J.R.; Davie, A.; Tocher, D.R. Biosynthesis of very long-chain fatty acids (C>24) in Atlantic salmon: Cloning, functional characterisation, and tissue distribution of an Elovl4 elongase. Comp. Biochem. Physiol. B 2011, 159, 122-129. [CrossRef] [PubMed]

18. Monroig, Ó.; Wang, S.; Zhang, L.; You, C.; Tocher, D.R.; Li, Y. Elongation of long-chain fatty acids in rabbitfish Siganus canaliculatus: Cloning, functional characterisation and tissue distribution of Elovl5- and Elovl4-like elongases. Aquaculture 2012, 350-353, 63-70. [CrossRef]

19. Li, S.; Monroig, Ó.; Navarro, J.C.; Yuan, Y.; Xu, W.; Mai, K.; Tocher, D.R.; Ai, Q. Molecular Cloning, functional characterization and nutritional regulation by dietary fatty acid profiles of a putative Elovl4 gene in orange-spotted grouper Epinephelus coioides. Aquac. Res. 2017, 48, 537-552. [CrossRef]

20. Monroig, Ó.; Hontoria, F.; Varó, I.; Tocher, D.R.; Navarro, J.C. Investigating the essential fatty acids in the common cuttlefish Sepia officinalis (Mollusca, Cephalopoda): Molecular cloning and functional characterisation of fatty acyl desaturase and elongase. Aquaculture 2016, 450, 38-47. [CrossRef]

21. Joseph, J.D. Lipid composition of marine and estuarine invertebrates. Part II: Mollusca. Prog. Lipid Res. 1982, 21, 109-153. [CrossRef]

22. Barnathan, G. Non-methylene-interrupted fatty acids from marine invertebrates: Occurrence, characterization and biological properties. Biochimie 2009, 91, 671-678. [CrossRef] [PubMed]

23. Kornprobst, J.M.; Barnathan, G. Demospongic acids revisited. Mar. Drugs 2010, 8, 2569-2577. [CrossRef] [PubMed]

24. Castro, L.F.C.; Wilson, J.M.; Gonçalves, O.; Galante-Oliveira, S.; Rocha, E.; Cunha, I. The evolutionary history of the stearoyl-CoA desaturase gene family in vertebrates. BMC Evol. 2011, 11, 132. [CrossRef] [PubMed]

25. Watts, J.L.; Browse, J. A palmitoyl-CoA-specific $\Delta 9$ fatty acid desaturase from Caenorhabditis elegans. Biochem. Biophys. Res. Commun. 2000, 272, 263-269. [CrossRef] [PubMed]

26. Jakobsson, A.; Westerberg, R.; Jacobsson, A. Fatty acid elongases in mammals: Their regulation and roles in metabolism. Prog. Lipid Res. 2006, 45, 237-249. [CrossRef] [PubMed]

27. Meyer, A.; Kirsch, H.; Domergue, F.; Abbadi, A.; Sperling, P.; Bauer, J.; Cirpus, P.; Zank, T.K.; Moreau, H.; Roscoe, T.J.; et al. Novel fatty acid elongases and their use for the reconstitution of docosahexaenoic acid biosynthesis. J. Lipid Res. 2004, 45, 1899-1909. [CrossRef] [PubMed]

28. Stukey, J.; McDonough, V.; Martin, C. Isolation and characterization of OLE1, a gene affecting fatty acid desaturation from Saccharomyces cerevisiae. J. Biol. Chem. 1989, 264, 16537-16544. [PubMed]

29. Porta, A.; Fortino, V.; Armenante, A.; Maresca, B. Cloning and characterization of a D9-desaturase gene of the Antarctic fish Chionodraco hamatus and Trematomus bernacchii. J. Comp. Physiol. B 2013, 183, 379-392. [CrossRef] [PubMed]

30. Agaba, M.; Tocher, D.R.; Dickson, C.; Dick, J.R.; Teale, A.J. Zebrafish cDNA encoding multifunctional fatty acid elongase involved in production of eicosapentaenoic (20:5n-3) and docosahexaenoic (22:6n-3) acids. Mar. Biotechnol. 2004, 6, 251-261. [CrossRef] [PubMed]

31. Bell, M.V.; Tocher, D.R. Biosynthesis of polyunsaturated fatty acids in aquatic ecosystems: General pathways and new directions. In Lipids in Aquatic Ecosystems; Arts, M.T., Brett, M., Kainz, M., Eds.; Springer: New York, NY, USA, 2009; pp. 211-236.

32. Tocher, D.R. Omega-3 long-chain polyunsaturated fatty acids and aquaculture in perspective. Aquaculture 2015, 449, 94-107. [CrossRef]

33. Reis, D.B.; Acosta, N.G.; Almansa, E.; Navarro, J.C.; Tocher, D.R.; Monroig, O.; Andrade, J.P.; Sykes, A.V.; Rodríguez, C. In vivo metabolism of unsaturated fatty acids in Octopus vulgaris hatchlings determined by incubation with ${ }^{14} \mathrm{C}$-labelled fatty acids added directly to seawater as protein complexes. Aquaculture 2014, 431, 28-33. [CrossRef]

34. Agbaga, M.-P.; Brush, R.S.; Mandal, M.N.A.; Henry, K.; Elliott, M.H.; Anderson, R.E. Role of Stargardt-3 macular dystrophy protein (ELOVL4) in the biosynthesis of very long chain fatty acids. Proc. Natl. Acad. Sci. USA 2008, 105, 12843-12848. [CrossRef] [PubMed] 
35. Albertin, C.B.; Simakov, O.; Mitros, T.; Wang, Z.Y.; Pungor, J.R.; Edsinger-Gonzales, E.; Brenner, S.; Ragsdale, C.W.; Rokhsar, D.S. The octopus genome and the evolution of cephalopod neural and morphological novelties. Nature 2015, 524, 220-224. [CrossRef] [PubMed]

36. Liu, H.; Guo, Z.; Zheng, H.; Wang, S.; Wang, Y.; Liu, W.; Zhang, G. Functional characterization of a $\Delta 5$-like fatty acyl desaturase and its expression during early embryogenesis in the noble scallop Chlamys nobilis Reeve. Mol. Biol. Rep. 2014, 41, 7437-7445. [CrossRef] [PubMed]

37. Li, M.; Mai, K.; He, G.; Ai, Q.; Zhang, W.; Xu, W.; Wang, J.; Liufu, Z.; Zhang, Y.; Zhou, H. Characterization of $\Delta 5$ fatty acyl desaturase in abalone Haliotis discus hannai Ino. Aquaculture 2013, 416-417, 48-56. [CrossRef]

38. Surm, J.M.; Prentis, P.J.; Pavasovic, A. Comparative analysis and distribution of Omega-3 LC-PUFA biosynthesis genes in marine molluscs. PLoS ONE 2015, 10, e0136301. [CrossRef] [PubMed]

39. Liu, H.; Zang, H.; Zheng, H.; Wang, S.; Guo, Z.; Zhang, G. PUFA Biosynthesis pathway in marine scallop Chlamys nobilis Reeve. J. Agric. Food Chem. 2014, 62, 12384-12391. [CrossRef] [PubMed]

40. Agbaga, M.-P.; Mandal, M.N.A.; Anderson, R.E. Retinal very long-chain PUFAs: New insights from studies on ELOVL4 protein. J. Lipid Res. 2010, 51, 1624-1642. [CrossRef] [PubMed]

41. McMahon, A.; Jackson, S.N.; Woods, A.S.; Kedzierski, W. A Stargardt disease-3 mutation in the mouse Elovl4 gene causes retinal deficiency of $\mathrm{C}_{32}-\mathrm{C}_{36}$ acyl phosphatidylcholines. FEBS Lett. 2007, 581, 5459-5463. [CrossRef] [PubMed]

42. Furland, N.E.; Oresti, G.M.; Antollini, S.S.; Venturino, A.; Maldonado, E.N.; Aveldaño, M.I. Very long-chain polyunsaturated fatty acids are the major acyl groups of sphingomyelins and ceramides in the head of mammalian spermatozoa. J. Biol. Chem. 2007, 282, 18151-18161. [CrossRef] [PubMed]

43. Zadravec, D.; Tvrdik, P.; Guillou, H.; Haslam, R.; Kobayashi, T.; Napier, J.A.; Capecchi, M.R.; Jacobsson, A. ELOVL2 controls the level of $n-6$ 28:5 and 30:5 fatty acids in testis, a prerequisite for male fertility and sperm maturation in mice. J. Lipid Res. 2011, 52, 245-255. [CrossRef] [PubMed]

44. Zhukova, N.V. Lipid classes and fatty acid composition of the tropical nudibranch mollusks Chromodoris sp. and Phyllidia coelestis. Lipids 2007, 42, 1169-1175. [CrossRef] [PubMed]

45. Zhukova, N.V. Lipids and fatty acids of nudibranch mollusks: Potential sources of bioactive compounds. Mar. Drugs 2014, 12, 4578-4592. [CrossRef] [PubMed]

46. Hastings, N.; Agaba, M.; Tocher, D.R.; Leaver, M.J.; Dick, J.R.; Sargent, J.R.; Teale, A.J. A vertebrate fatty acid desaturase with $\Delta 5$ and $\Delta 6$ activities. Proc. Natl. Acad. Sci. USA 2001, 98, 14304-14309. [CrossRef] [PubMed]

47. Lopes-Marques, M.; Ozório, R.; Amaral, R.; Tocher, D.R.; Monroig, Ó.; Castro, L.F.C. Molecular and functional characterization of a fads 2 orthologue in the Amazonian teleost, Arapaima gigas. Comp. Biochem. Physiol. B 2017, 203, 84-91. [CrossRef] [PubMed]

48. Folch, J.; Lees, N.; Sloane-Stanley, G.H. A simple method for the isolation and purification of total lipids from animal tissues. J. Biol. Chem. 1957, 226, 497-509. [PubMed]

49. Destaillats, F.; Angers, P. One-step methodology for the synthesis of FA picolinyl esters from intact lipids. J. Am. Oil Chem. Soc. 2002, 79, 253-256. [CrossRef]

50. Li, Y.; Monroig, Ó.; Zhang, L.; Wang, S.; Zheng, X.; Dick, J.R.; You, C.; Tocher, D.R. Vertebrate fatty acyl desaturase with $\Delta 4$ activity. Proc. Natl. Acad. Sci. USA 2010, 107, 16840-16845. [CrossRef] [PubMed]

(C) 2017 by the authors. Licensee MDPI, Basel, Switzerland. This article is an open access article distributed under the terms and conditions of the Creative Commons Attribution (CC BY) license (http:/ / creativecommons.org/licenses/by/4.0/). 